\title{
Ovário-histerectomia: estudo experimental comparativo entre as abordagens laparoscópica e aberta na espécie canina- III. Estresse pela análise do cortisol plasmático
}

[Ovariohysterectomy: experimental and comparative study between laparoscopic and conventional approachesIII. Stress by plasmatic cortisol analysis]

\author{
C. Malm ${ }^{1}$, P.R. Savassi-Rocha ${ }^{2}$, V.A. Gheller ${ }^{1}$, H.P. Oliveira ${ }^{1}$, A.R. Lamounier ${ }^{3}$, V. Foltynek ${ }^{4}$ \\ ${ }^{1}$ Departamento de Clínica e Cirurgia Veterinárias - Escola de Veterinária da UFMG \\ Caixa Postal 567 \\ 30123-970 - Belo Horizonte, MG \\ ${ }^{2}$ Instituto Alfa de Gastroenterologia - Hospital das Clínicas da UFMG \\ ${ }^{3}$ Escola de Veterinária - PUC Betim \\ ${ }^{4}$ Estudante de Mestrado - EVUFMG
}

\begin{abstract}
RESUMO
Avaliou-se o estresse pós-operatório, durante sete dias, em 30 cadelas, aleatoriamente distribuídas em dois grupos de 15 animais submetidos à ovário-histerectomia $(\mathrm{OVH})$ pelas abordagens laparoscópica (grupo I) e aberta (grupo II). Os grupos foram comparados mediante dosagens do cortisol plasmático. Não se observou aumento do cortisol plasmático no pré-operatório imediato (tempo 1) e quando o animal estava sob anestesia geral imediatamente antes do procedimento cirúrgico (tempo 2). Nos dois grupos houve aumento do cortisol apenas no período intra-operatório (tempo 3) e uma hora após o retorno anestésico (tempo 4), sendo maior nas cadelas submetidas à OVH por laparoscopia. No pós-operatório, os níveis de cortisol das cadelas de ambos os grupos apresentaram-se normais e equivalentes aos do préoperatório. Independente da abordagem utilizada, a resposta ao estresse foi semelhante para os dois grupos.
\end{abstract}

Palavras-chave: cadela, laparoscopia, ovário-histerectomia, estresse, cortisol

\begin{abstract}
Post-operative stress, as indicated by changes in plasma cortisol concentration, was monitored for seven days in 30 bitches, half of whom were subjected to conventional (group I) and half to laparoscopic ovariohysterectomy (group II). No increases on plasmatic cortisol concentration were observed in the pre-surgery phase (time 1) and when animals were under anaesthesia just before surgery (time 2). Cortisol concentration increased in both groups only during the intra-operative period (time 3) and one hour after recovery from anesthesia (time 4). Cortisol levels were higher in the bitches submitted to laparoscopy than in those experiencing conventional surgery. For both surgical approaches, cortisol concentrations in the post-operative phase returned to values characteristic of the pre-operative phase. Response to stress, assessed from changes in plasma cortisol concentration, was similar for both groups.
\end{abstract}

Keywords: bitch, ovariohysterectomy, laparoscopy, stress, cortisol

Recebido para publicação em 13 de julho de 2004

Recebido para publicação, após modificações, em 25 de maio de 2005

E-mail: malm@vet.ufmg.br 


\section{INTRODUÇÃO}

A dor, o estresse e o sofrimento ameaçam o bemestar do animal e, eventualmente, sua sobrevivência. Muitas vezes, ele apresenta mudanças de comportamento na tentativa de aliviar uma condição de dor e ameaça. Quando essas respostas são insuficientes para aliviar o estresse, o sistema nervoso autônomo e neuroendócrino são ativados, acarretando alterações em vários parâmetros físiológicos e bioquímicos (Moberg, 1987; Introduction..., 1992; Recognition..., 1992), comumente referidas como resposta ao estresse (Kehlet, 1989).

Respostas do sistema nervoso autônomo (SNA), como taquicardia, taquipnéia, aumento da pressão arterial, arritmias, salivação, midríase, sudorese e liberação de catecolaminas são indicativas de dor e estresse, principalmente quando estão associadas às alterações do comportamento. Devido à rápida e específica resposta do SNA a determinados agentes estressores, as mensurações das freqüências cardíaca e respiratória e a secreção de catecolaminas podem ser utilizadas na avaliação do estresse. No entanto, devido à fugaz $\mathrm{e}$ inconstante resposta desses parâmetros, essas mensurações mostram-se difíceis e pouco confiáveis (Moberg, 1987; Sackman, 1991; Recognition..., 1992).

Situações estressantes e dolorosas podem alterar a secreção dos hormônios hipofisários que regulam diretamente as funções relacionadas ao bem-estar do animal como reprodução, crescimento e resistência imunológica. Dentre os hormônios hipofisários, o adrenocorticotrópico (ACTH) estimula a secreção de corticosteróides em condições de estresse (Moberg, 1987; Sackman, 1991; Recognition..., 1992). O cortisol é um parâmetro preciso e consistente para avaliação da resposta neuroendócrina ao estresse cirúrgico, indicando a presença da dor (Day et al., 1995; Smith et al, 1996).

O trauma cirúrgico e a dor pós-operatória podem provocar a ativação neuroendócrina e metabólica com conseqüente hipermetabolismo, aceleração de reações bioquímicas e catabolismo orgânico. A duração e a intensidade das alterações estão relacionadas com o grau da lesão tissular e podem prolongar o período de convalescença e de recuperação pós-operatória. Os procedimentos cirúrgicos minimamente invasivos podem evitar ou diminuir as alterações fisiológicas e endócrino-metabólicas (Bonica, 1992).

Na medicina humana, as cirurgias com mínimo trauma oferecem vantagens quando comparadas com as convencionais. Pequenas incisões e menor trauma tissular podem resultar em menos dor pós-operatória, melhor função respiratória, menor tempo de hospitalização e recuperação pós-operatória mais rápida (Frazee et al., 1991; Graves et al., 1991; Savassi-Rocha, 1994).

Na medicina veterinária, a cirurgia laparoscópica constitui uma abordagem inovadora que tem sido utilizada em diversos procedimentos cirúrgicos. No entanto, não existem estudos clínicos comparativos entre as abordagens laparoscópica e aberta que avaliem a dor pós-operatória e a recuperação do animal nesta fase.

Considerando a subjetividade e a dificuldade no reconhecimento da dor em animais, parâmetros comportamentais, fisiológicos e hormonais devem ser analisados em conjunto, resultando em avaliações mais precisas da dor e da recuperação pós-operatória do paciente (Agnati et al., 1991; Mathews, 2000).

No acompanhamento do pós-operatório e do reconhecimento da dor, avaliações clínicas, de comportamento e complicações na ferida cirúrgica foram estudadas e descritas por Malm et al. (2005).

Este trabalho teve como objetivo mensurar o cortisol plasmático nos períodos pré, trans e pós operatórios, bem como avaliar o estresse pósoperatório de cadelas submetidas à ováriohisterectomia pelas abordagens laparoscópica e aberta, através da mensuração do cortisol plasmático.

\section{MATERIAL E MÉTODOS}

Foram utilizadas 30 cadelas, hígidas, sem raça definida, jovens (oito meses a dois anos de idade) pesando entre 6,5 e $19,0 \mathrm{~kg}$. Os animais permaneceram, durante 15 dias, em canis com solário para fins de observação e adaptação, 
sendo alimentados com ração comercial ${ }^{1}$. Em seguida, foram alojados em canis individuais e distribuídos ao acaso, formando dois grupos de 15.

No pré-operatório imediato, procedeu-se à administração de cefalotina sódica ${ }^{2}$ (30mg/kg/IV), 30 minutos antes da intervenção cirúrgica. $\mathrm{O}$ preparo do equipamento laparoscópico para os procedimentos cirúrgicos, a esterilização do material e a montagem da equipe cirúrgica foram descritos por Malm et al. (2004). Os animais foram submetidos aos procedimentos pré-anestésico e anestésico e, em seguida, à $\mathrm{OVH}$ laparoscópica (grupo I) e $\mathrm{OVH}$ aberta (grupo II), segundo Malm et al. (2004). Logo após o término do procedimento cirúrgico, procedeu-se ao curativo das feridas cirúrgicas com gaze estéril e esparadrapo e à colocação do colar elisabetano. Não foram administradas drogas analgésicas no pós-operatório, para que não houvesse qualquer interferência nas avaliações hormonais propostas. Os animais permaneceram em canis individuais durante $\mathrm{o}$ retorno anestésico.

Foram colhidas amostras de $5,0 \mathrm{ml}$ de sangue por venopunção jugular, utilizando sistema de coleta de sangue a vácuo ${ }^{3}$ com agulhas hipodérmicas $25 \times 8 \mathrm{~mm}^{4}$, para a dosagem do cortisol plasmático nos seguintes tempos: T1 - no pré-operatório imediato, com o animal ainda no canil; T2 - com o animal sob anestesia geral, imediatamente antes de iniciar o procedimento cirúrgico; T3 durante a manipulação intra-operatória do pedículo ovariano direito ou esquerdo; T4 - uma hora após o retorno da anestesia; T5 a T11 - uma vez ao dia entre oito e 12 horas da manhã, durante os sete dias de pós-operatório, sendo o (T5) realizado 24 horas após a cirurgia.

As amostras de sangue foram centrifugadas ${ }^{5}$ a $2.000 \mathrm{rpm}$ durante 10 minutos, e o plasma obtido acondicionado em ependorfes, devidamente identificados em duplicatas e mantidos a $-20{ }^{\circ} \mathrm{C}$,

\footnotetext{
${ }^{1}$ Herói - GUABI - Mogiana Alimentos S.A, Campinas, Brasil

${ }^{2}$ Cefalotina - ARISTON - Indústria Química e Farmacêutica Ltda., São Paulo, Brasil

${ }^{3}$ Tubo vacuntainer com EDTA K3E, BD- Becton Dickinson and Company, USA

4 Agulha para coleta de sangue a vácuo, BD- Becton Dickinson and Company, USA

${ }^{5}$ Centrifugadora Excelsa Baby I, Mod. 208N, FANEM, São Paulo, Brasil
}

em congelador horizontal, para posterior processamento. A concentração plasmática do cortisol foi dosada, em duplicata, mediante técnica de radioimunoensaio de fase sólida, utilizando kit comercial $^{6}$.

As cadelas foram as mesmas utilizadas por Malm et al. (2005), que avaliaram a evolução clínica e a dor pós-operatória mediante parâmetros de comportamento, fisiológicos e complicações na ferida cirúrgica.

Os resultados foram submetidos à análise de variância, com médias comparadas pelo teste Duncan. As diferenças foram consideradas significativas quando $\mathrm{P}<0,05$.

\section{RESULTADOS}

Não se observou aumento do cortisol plamático no pré-operatório imediato (T1) e quando o animal estava sob anestesia geral, imediatamente antes do procedimento cirúrgico (T2). A concentração do cortisol plasmático aumentou apenas no intra-operatório (T3) e uma hora após o retorno da anestesia (T4) nas duas abordagens cirúrgicas. Quando os dois grupos foram comparados entre si, observaram-se diferenças significativas em T3 $(p=0,0001)$ e T4 ( $p=$ $0,0023)$, com níveis mais elevados nos animais operados pela abordagem laparoscópica (Fig. 1).

Nos dois grupos experimentais, durante o pósoperatório (T5 a T11), a concentração de cortisol diminuiu para níveis normais e semelhantes aos do pré-operatório (Fig. 1). As médias dos valores de cortisol ultrapassaram aqueles admitidos para a espécie canina apenas em T3 e T4 no grupo I, e em T4 no grupo II (Tab. 1).

\section{DISCUSSÃO}

A OVH, procedimento cirúrgico rotineiramente realizado na clínica cirúrgica de pequenos animais, provoca dor pós-operatória de intensidade leve a moderada em razão de fatores como duração e extensão da cirurgia, grau de manipulação, idade e escore corporal do animal (Mathews, 2000). A dor é uma condição

\footnotetext{
${ }^{6}$ Cortisol Coat-A-Count, DPC MEDLAB Produtos MédicoHospitalares LTDA., São Paulo, Brasil.
} 
universal que, apesar de comumente experimentada, é de difícil avaliação (Hall, 2000), sendo também fortemente influenciada pela individualidade e por elementos emocionais, como medo, excitação, ansiedade e experiências dolorosas vividas anteriormente (Sackman, 1991; Wall, 1992; Hardie, 2002).

Apesar de se avaliar a dor e o estresse animal mediante critérios de comportamento (Hansen, 1997; Hardie et al., 1997; Hardie, 2002), esses parâmetros devem ser associados a índices fisiológicos e respostas endócrino-metabólicas que permitem interpretações clínicas mais precisas (Agnati et al., 1991; Johnson, 1991; Hansen et al., 1997; Mathews, 2000). A mensuração do cortisol é utilizada para avaliar a resposta do eixo hipotálamo-hipófise-adrenais em procedimentos anestésicos e cirúrgicos tanto no homem como nos animais (Hashimoto e Migita, 1979; Bonica, 1992; Fox et al., 1994,1998) e tem-se mostrado um método exeqüível e confiável para reconhecimento da dor nos animais (Smith et al, 1996; Fox et al., 1994, 1998).

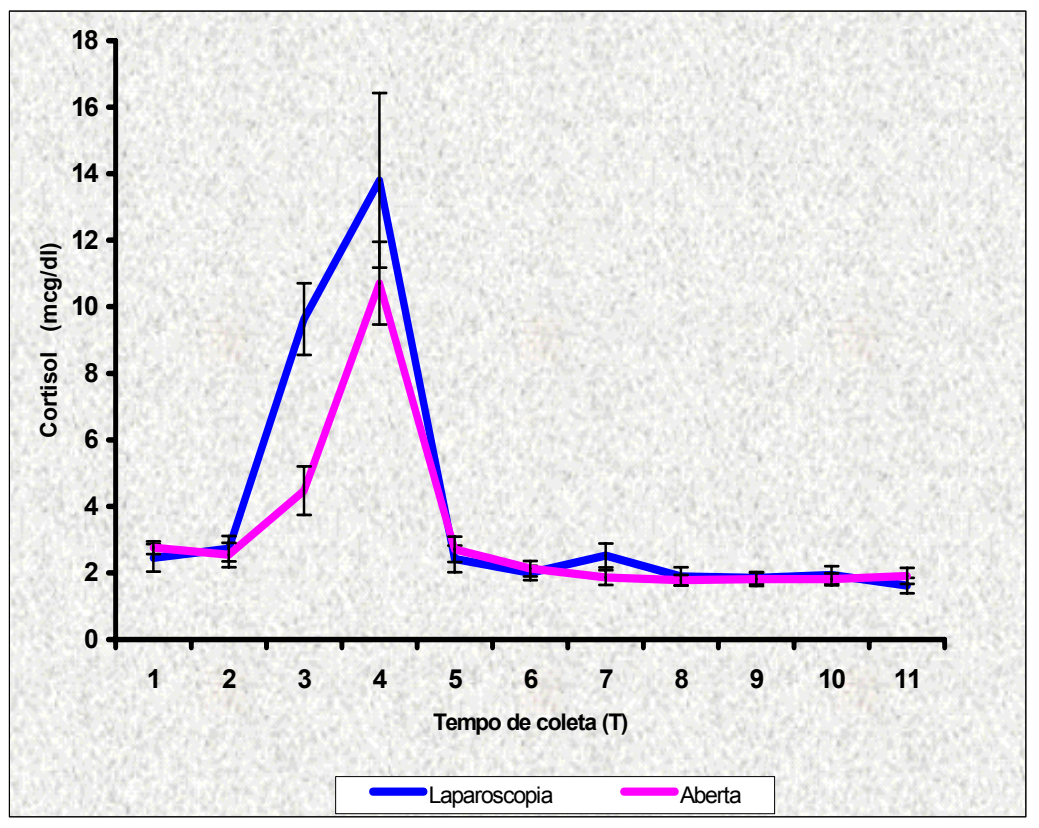

Figura 1. Valores médios de cortisol plasmático $(\mathrm{mcg} / \mathrm{dl})$, em diferentes tempos de coletas em cadelas submetidas à ovário-histerectomia por laparoscopia (grupo I) e cirurgia aberta (grupo II) (teste de Duncan). Tempos: $\mathrm{T} 1=$ pré-operatório; $\mathrm{T} 2=$ animal sob anestesia geral; $\mathrm{T} 3=$ intra-operatório; $\mathrm{T} 4=$ uma hora após o retorno da anestesia; T5 a T11= pós-operatório, sendo as coletas realizadas uma vez ao dia, durante sete dias.

No presente estudo, em ambos os grupos, houve elevação da concentração do cortisol apenas no período intra-operatório e após o retorno anestésico. Smith et al. (1996) e Fox et al. (1994, 1998) verificaram elevação desse hormônio em gatas e cadelas durante a $\mathrm{OVH}$ e durante a recuperação anestésica. A elevação desse hormônio durante a cirurgia deve-se, provavelmente, aos estímulos nociceptivos. $\mathrm{Na}$ recuperação anestésica, pode ser atribuída à combinação do efeito residual dos estímulos nociceptivos cirúrgicos com o retorno da consciência do animal (Fox et al., 1994, 1998). As maiores concentrações de cortisol ocorreram no tempo 3 (uma hora após o retorno anestésico). Deve-se considerar que o cloridrato de xilazina utilizado como droga pré-anestésica apresenta ações sedativa, miorrelaxante e analgésica por apenas 20 a 40 minutos (Muir et al., 1995). Assim, esse fármaco, pelo tempo de ação analgésica citada, não sugere interferência nos níveis de cortisol encontrados durante a fase de 
recuperação pós-anestésica. É importante salientar que não foram administradas outras drogas analgésicas no pós-operatório para que não houvesse qualquer interferência nas avaliações do cortisol.

Tabela 1. Valores médios de cortisol plasmático $\left(\mathrm{mcg} / \mathrm{dl}{ }^{(*)}\right)$, em diferentes tempos de coletas sangüíneas em cadelas submetidas à ováriohisterectomia por laparoscopia (grupo I) e cirurgia aberta (grupo II)

\begin{tabular}{lcc}
\hline Tempo $^{\left({ }^{*}\right)}$ & Laparoscopia (grupo I) & Aberta (grupo II) \\
\hline T1 & $2,45 \pm 0,41$ a A & $2,76 \pm 0,19$ a,c A \\
T2 & $2,73 \pm 0,38$ a A & $2,54 \pm 0,37$ a,c A \\
T3 & $\mathbf{9 , 6 3} \pm \mathbf{1 , 0 8}$ b A & $4,47 \pm 0,73$ c B \\
T4 & $\mathbf{1 3 , 8} \pm \mathbf{2 , 6 2}$ c A & $\mathbf{1 0 , 7 1} \pm \mathbf{1 , 2 4}$ b B \\
T5 & $2,42 \pm 0,40$ a A & $2,71 \pm 0,38$ a,c A \\
T6 & $2,00 \pm 0,22$ a A & $2,13 \pm 0,23$ a A \\
T7 & $2,52 \pm 0,36$ a A & $1,86 \pm 0,22$ a A \\
T8 & $1,90 \pm 0,27$ a A & $1,78 \pm 0,16$ a A \\
T9 & $1,85 \pm 0,18$ a A & $1,81 \pm 0,20$ a A \\
T10 & $1,94 \pm 0,27$ a A & $1,81 \pm 0,18$ a A \\
T11 & $1,62 \pm 0,23$ a A & $1,81 \pm 0,24$ a A \\
\hline
\end{tabular}

Médias seguidas por letras minúsculas distintas na mesma coluna diferem entre si $(\mathrm{P}<0,05)$.

Médias seguidas por letras maiúsculas distintas na mesma linha diferem entre si $(\mathrm{P}<0,05)$.

${ }^{(*)}$ Valores de referência para a espécie canina: 0,7 a $6 \mu \mathrm{g} / \mathrm{dl}$ (Bush, 1991).

${ }^{(* *)} \mathrm{T} 1=$ pré-operatório; $\mathrm{T} 2=$ animal sob anestesia geral; $\mathrm{T} 3=$ intra-operatório; T4= uma hora após o retorno da anestesia; T5 a T11= pós-operatório, sendo as coletas realizadas uma vez ao dia, durante sete dias.

Os valores de cortisol nos tempos 3 e 4 apresentaram-se mais elevados nas cadelas operadas por laparoscopia em relação àquelas submetidas às cirurgias abertas, o que pode ser atribuído ao maior tempo de manipulação dos tecidos e órgãos. Os tempos cirúrgicos no grupo I foram comparativamente mais longos $(61,6 \pm$ 14,1 minutos) do que aqueles do grupo II $(21,1 \pm$ 4,3 minutos), conforme descrito por Malm et al. (2004). Esse tempo cirúrgico mais longo nas cirurgias laparoscópicas deveu-se às manobras de tração, dissecção, clipagem e secção dos pedículos ovarianos, bem como à aplicação das endoloopes no corpo uterino. Segundo Fox et al. (1994, 1998) e Ko et al. (2000), as manobras cirúrgicas ovarianas provocam estímulo nociceptivo significativo, com elevação nos níveis de cortisol plasmático. Clarke et al. (1970), Madsen et al. (1976), Schmidt e Booker (1982) e Chernow et al. (1987) comentaram que a elevação no cortisol está associada à intensidade do trauma cirúrgico e que é mais elevada em cirurgias abdominais mais extensas, quando comparadas com procedimentos menores ou superficiais. Apesar de a cirurgia laparoscópica ser um procedimento menos invasivo, o tempo cirúrgico elevado, as alterações cardiocirculatórias, pulmonares e hemodinâmicas resultantes do pneumoperitônio e a maior manipulação do útero e ovários podem estar relacionadas com os valores mais elevados de cortisol no intra-operatório encontrados no grupo I deste estudo.

Neste trabalho, não foram observadas elevações do cortisol plasmático enquanto os animais estavam anestesiados e antes da intervenção cirúrgica. Clarke et al. (1970), Church et al. (1994), Fox et al. (1994) e Hansen et al. (1997) comentaram que os níveis de cortisol podem elevar durante os momentos de indução anestésica, excitação e intubação orotraqueal, podem diminuir durante a anestesia e elevar novamente no intra-operatório. $\mathrm{O}$ fármaco préanestésico utilizado contribuiu para a indução anestésica sem excitação, não comprometendo, em princípio, os níveis de cortisol nessa fase.

A concentração de cortisol em cadelas aumenta significativamente durante a manipulação dos ovários e permanece elevada após o término do procedimento cirúrgico (Fox et al., 1998; Ko et al., 2000), a exemplo do que se verificou nesta pesquisa. Em ambos os grupos, houve aumento do cortisol no intra-operatório (T3) e uma hora após o retorno anestésico (T4). Este último tempo correspondeu à coleta de sangue realizada em um período que variou entre uma hora e 15 minutos a quatro horas após o término do procedimento cirúrgico. Hansen (1997) comentou que a dor pós-operatória apresenta um curso previsto, com pico entre seis e 24 horas após a cirurgia e diminuição progressiva.

A partir do primeiro dia de pós-operatório ( 24 horas após a cirurgia), os níveis de cortisol retornaram aos valores considerados normais para a espécie canina e permaneceram inalterados durante os sete dias estudados. Podese considerar que as condições de dor e de estresse foram pouco significativas durante este período tanto nas cadelas operadas pela abordagem laparoscópica como pela aberta, já que os valores do cortisol permaneceram em 
níveis basais durante todo o período de avaliação pós-operatória nesses animais que não receberam analgesia. Neste estudo, não foi mensurado o cortisol em outros tempos (entre quatro e 24 horas após a cirurgia), mas deve-se considerar que, segundo Hansen et al. (1997) e Hardie et al. (1997), cadelas ovário-histerectomizadas, sem analgesia, apresentam concentrações elevadas de cortisol entre três e 12 horas após a cirurgia, bem como alterações de comportamento marcantes por, pelo menos, 24 horas, indicando ser a $\mathrm{OVH}$ uma experiência estressante. Em cães submetidos a diferentes intervenções cirúrgicas, com e sem analgesia, as concentrações de cortisol retornam ao normal em até 24 horas (Church et al., 1994; Fox et al., 1994), indicando que os estímulos nociceptivos cirúrgicos aliados aos fatores psicogênicos são significativos e desaparecem parcialmente entre cinco e 24 horas após a intervenção.

É altamente recomendado o uso de analgésicos em procedimentos que promovam a dor pósoperatória, mesmo que não haja um evidente reconhecimento das alterações de comportamento exibidas pelo animal (Cozemius et al., 1997; Ko et al., 2000). Deve-se considerar que a dor e o estresse podem interferir na convalescença e na recuperação pós-operatória do paciente (Bonica, 1992).

\section{CONCLUSÕES}

Os níveis do cortisol plasmático em cadelas submetidas à $\mathrm{OVH}$ pelas abordagens laparoscópica e aberta elevam-se significativamente no intra-operatório durante manobras dos pedículos ovarianos e no pósoperatório imediato (uma hora após o retorno da anestesia). Os níveis do cortisol plasmático evidenciam que, durante sete dias do pósoperatório, o estresse mostra-se semelhante para as duas abordagens cirúrgicas estudadas.

\section{REFERÊNCIAS BIBLIOGRÁFICAS}

AGNATI, L.F.; TIENGO, M.; FERRAGUTI, F. et al. Pain, analgesia, and stress: an integrated view. Clin. J. Pain, v.7, suppl.1, p.23-37, 1991.

BONICA, J.J. Pain research and therapy: history, current status and future goals. In: SHORT, C.E.;
POZNACK, A.V. (Eds.). Animal pain. New York: Churchill Livingstone, 1992. cap.1, p.129.

CHERNOW, B.; ALEXANDER, H.R.; SMALLRIDGE, R.C. et al. Hormonal responses to graded surgical stress. Arch. Intern. Med., v.147, p.1273-1278, 1987.

CHURCH, D.B.; NICHOLSON, A.I.; ILKIW, J.E. et al. Effect of non-adrenal illness, anaesthesia and surgery on plasma cortisol concentrations in dogs. Res. Vet. Sci., v.56, p.129-131, 1994.

CLARKE, R.S.J.; JOHNSTON, H.; SHERIDAN, B. The influence of anaesthesia and surgery on plasma cortisol, insulin and free fatty acids. Br. J. Anaesth., v.42, p.295-299, 1970.

CONZEMIUS, M.G.; HILL, C.M.; SQAMMARCO, J.L. et al. Correlation between subjective and objective measures used to determine severity of postoperative pain in dogs. J. Am. Vet. Med. Assoc., v. 210, p.1619-1622, 1997.

DAY, T.K.; PEPPER, W.T.; TOBIAS, T.A. et al. Comparison of intra-articular and epidural morphine for analgesia following stifle arthrotomy in dogs. Vet. Surg., v.24, p.522-530, 1995.

FOX, S.M.; D.J., MELLOR, D.J.; FIRTH, E.C. et al. Changes in plasma cortisol concentrations before, during and after analgesia, anesthesia plus ovariohysterectomy in bitches. Res. Vet. Sci., v.57, p.110-118, 1994.

FOX, S.M.; D.J., MELLOR, D.J.; LAWOKO, C.R.O. et al. Changes in plasma cortisol concentrations in bitches in response to different combinations of halotane and butorphanol with or without ovariohysterectomy. Res. Vet. Sci., v.65, p.125-133, 1998.

FRAZEE, M.D.; ROBERTS, J.W.; OKESON, G.C. et al. Open versus laparoscopic cholecystectomy - A comparison of postoperative pulmonary function. Ann. Surg., v.213, p.651-653, 1991.

GRAVES, H.A.; BALLINGER, J.F.; ANDERSON, W.J. Appraisal of laparoscopic cholecystectomy. Ann. Surg., v.213, p.655-664, 1991.

HALL, J. The nature of pain. Nurs. Time, v. 96, p.37-40, 2000. 
HANSEN, B. Through a glass darkly: using behaviour to assess pain. Semin. Vet. Med. Surg.: Small Anim., v.12, p.61-74, 1997.

HANSEN, B.D.; HARDIE, E.M.; CARROL, G.S. Physiological measurements after ovariohysterectomy in dogs: what's normal? Appl. Anim. Behav. Sci., v. 51, p.101-109, 1997.

HARDIE, E.M. Reconhecimento do comportamento doloroso em animais. In: HELLEBREKERS, L.J. Dor em animais: uma abordagem com orientação prática para um controle eficaz da dor em animais. São Paulo: Manole, 2002. cap.4, p.49-68.

HARDIE, E.M.; HANSEN, B.D.; CARROLL, G.S. Behavior after ovariohysterectomy in the dog: what's normal? Appl. Anim. Behav. Sci., v.51, p.111-128, 1997.

HASHIMOTO, S.; MIGITA, S. Changes of thirtynine serum protein components following surgical stress. Acta Haem. Jap., v.42, p.47-57, 1979.

INTRODUCTION. In: Recognition and alleviation of pain and distress in laboratory animals. Washington: National Academic, 1992. cap.1. Disponível em: <http://books.nap.edu/ books $/ 0309042755 / \mathrm{html} / \mathrm{R} 1 . \mathrm{html}>$. Acessado em 04/04/2003.

JOHNSON, J.M. The veterinarian's responsability assessing and managing acute pain in dogs and cats. Part I. Comp. Small Anim., v.13, p.804-807, 1991.

KEHLET, H. Surgical stress: the role of pain and analgesia. Br. J. Anaesth., v.63, p.189-195, 1989.

KO, J.C.H.; MANDSAGER, R.E.; LANGE, D.N. et al. Cardiorespiratory responses and plasma cortisol concentrations in dogs treated with medetomidine before undergoing ovariohysterectomy. J. Am. Vet. Med. Assoc., v.217, p.509-514, 2000.

MADSEN, S.N.; ENGQUIST, A.; BADAWI, I. et al. Cyclic AMP, glucose and cortisol in plasma during surgery. Horm. Metab. Res., v.8, p.483485,1976

MALM, C.; SAVASSI-ROCHA, P.R.; GHELLER, V.A. et al. Ovário-histerectomia: estudo experimental comparativo entre as abordagens laparoscópica e aberta na espécie canina. Intra-operatório-I. Arq. Bras. Med. Vet. Zootec., v.56, p.457-466, 2004.

MALM, C.; SAVASSI-ROCHA, P.R.; GHELLER, V.A. et al. Ovário-histerectomia: estudo experimento comparativo entre as abordagens laparoscópica e aberta na espécie canina. II- Evolução clínica pos-operatória. Arq. Bras. Med. Vet. Zootec., v.57, p.162-172, 2005.

MATHEWS, K.A. Pain assessment and general approach to management. Vet. Clin. North Am.: Small Anim. Pract., v.30, p.729-755, 2000.

MOBERG, G.P. Problems in defining stress and distress in animals. J. Am. Vet. Med. Assoc., v.191, p.1207-1211, 1987.

MUIR, W.W.; HUBBELL, J.AE.; SKARDA, R.T. et al. Handbook of veterinary anesthesia. 2.ed. St. Louis: Mosby-Year Book, 1995. 510 p.

RECOGNITION and assessment of pain, stress and distress. In: Recognition and alleviation of pain and distress in laboratory animals. Washington: National Academic, 1992. cap.4. Disponível em: <http://books.nap.edu/books/ 0309042755/html $/ \mathrm{R} 1 . \mathrm{html}>$. Acessado em 04/04/2003.

SACKMAN, J.E. Pain: its perception and alleviation in dogs and cats. Part I. The physiology of pain. Comp. Small Anim., v.13, p.71-75, 1991.

SAVASSI-ROCHA, P.R. Colecistectomia videolaparoscópica: um novo padrão ouro? In: CASTRO, L.P.; SAVASSI-ROCHA, P.R.; CUNHA-MELO, J.R. Tópicos em gastroenterologia. 5. Rio de Janeiro: Medsi, 1994. cap. 26, p.465-489.

SCHMIDT, R.E.; BOOKER, J.L. Effects of different surgical stresses on hematological and blood chemistry values in dogs. J. Am. Anim. Hosp. Assoc., v.18, p.758-762, 1982.

SMITH, J.D.; ALLEN, S.W.; QUANDT, J.E. et al. Indicators of postoperative pain in cats and correlation with clinical criteria. Am. J.Vet. Res., v.57, p.1674-1678, 1996.

WALL, P.D. Defining pain in animals. In: SHORT, C.E.; POZNAK, A.V. (Eds.). Animal pain. New York: Churchill Livingstone, 1992. cap. 3, p.63-79. 